\title{
GENERATING GROUPS OF CERTAIN SOLUBLE VARIETIES
}

\author{
Dedicated to the memory of Hanna Neumann \\ NARAIN GUPTA and FRANK LEVIN \\ (Received 21 June 1972, revised 20 December 1972) \\ Communicated by M. F. Newman
}

\section{Introduction}

Any variety of groups is generated by its free group of countably infinite rank. A problem that appears in various forms in Hanna Neumann's book [7] (see, for intance, sections $2.4,2.5,3.5,3.6$ ) is that of determining if a given variety $\mathfrak{B}$ can be generated by $F_{k}(\mathfrak{B})$, one of its free groups of finite rank; and if so, if $F_{n}(\mathfrak{B})$ is residually a $k$-generator group for all $n \geqq k$. (Here, as in the sequel, all unexplained notation follows [7].)

To any variety $\mathfrak{B}$ generated by a finitely generated group one can associate the number $d(\mathfrak{B})$, the least positive integer such that $\mathfrak{B}$ is generated by its free group of rank $d(\mathfrak{B})$. For example, for the variety $\mathfrak{D}$ of all groups, $d(\mathfrak{D})=2$ (in fact every free group is residually free of rank 2 [8]); for $\mathfrak{A}$, the variety of abelian groups, $d(\mathfrak{U})=1$ and $d\left(\mathfrak{H}^{l}\right)=2(l \geqq 2)([7] 16.35$ and 25.34$)$; for $\mathfrak{N}_{c}$, the variety of nilpotent groups of class at most $c, d\left(\mathfrak{N}_{c}\right)=c-1(c \geqq 3)([6],[9])$; and more generally for $\mathfrak{B} \leqq \mathfrak{N}_{c}, d(\mathfrak{B}) \leqq c([7] 35.12)$. Further examples may be found in [7] where, in addition, for two varieties $\mathfrak{U}$ and $\mathfrak{B}$, the dependence of $d(\mathfrak{U} \mathfrak{B})$ on $d(\mathfrak{U})$ and $d(\mathfrak{B})$ is discussed. Also, Baumslag [2] has shown that for arbitrary $\mathfrak{U}$, the non-cyclic free groups of $\mathfrak{U} \mathfrak{A}$ are residually free of rank 2 so that, in particular, $d(\mathfrak{U} \mathfrak{X}) \leqq 2$ (cf. [7] 25.33).

Corresponding results for $[\mathfrak{U}, \mathfrak{B}]$ are more isolated even for $\mathfrak{B}=\mathfrak{E}$, especially since $[\mathfrak{U}, \mathfrak{E}]$ is indecomposable for any $\mathfrak{U} \neq \mathfrak{D}([7] 24.32)$. In the present paper we shall consider such problems for $\mathfrak{M}_{(1)}=\left[\mathfrak{A}^{2}, \mathfrak{E}\right]$, the variety of centre-bymetabelian groups; and more generally for $\mathfrak{M}_{(c)}$, defined inductively by $\mathfrak{M}_{(c)}$ $=\left[\mathfrak{M}_{(c-1)}, \mathbb{E}\right](c \geqq 2)$. In addition, we obtain information regarding the ascending chains

$$
\operatorname{Var} F_{2}\left(\mathfrak{M}_{(c)}\right) \leqq \operatorname{Var} F_{3}\left(\mathfrak{M}_{(c)}\right) \leqq \cdots
$$

Research supported by grants from N. R. C. and N. S. F. respectively. 
(see [7] Section 1.6 for a general discussion of such chains).

Our results for $c=1,2$ rely heavily on a $3 \times 3$ matrix representation of $F_{\infty}\left(\mathfrak{M}_{(1)}\right)$ found by Gupta [4] and a corresponding $4 \times 4$ matrix representation of $F_{\infty}\left(\mathfrak{M}_{(2)}\right)$ (Section 4). These representations are generalizations of the well-known faithful $2 \times 2$ matrix representation of $F_{\infty}(\mathfrak{M})$ found by Magnus (see [7], 36.12), where $\mathfrak{M}=\mathfrak{U}^{2}$. In Section 2 we divert from our discussion to illustrate how the Magnus representation can be used to give an alternate and rather elementary proof of the result that $F_{k}(\mathfrak{M})$ is residually $F_{2}(\mathfrak{M})$ for $k \geqq 2$. On the whole, Section 2 serves the purpose of introducing the terminology and the computational techniques required in our discussion of $\mathfrak{M}_{(1)}$ and $\mathfrak{M}_{(2)}$.

In Section 3 we show that $d\left(\mathfrak{M}_{(1)}\right)=4$ (Theorem 3.7) and establish that

$$
\operatorname{Var} F_{2}\left(\mathfrak{M}_{(1)}\right)=\operatorname{Var} F_{3}\left(\mathfrak{M}_{(1)}\right)<\operatorname{Var} F_{4}\left(\mathfrak{M}_{(1)}\right)=\mathfrak{M}_{(1)}
$$

The inequality in (2) is a result of Gupta [5] who shows that the laws of $F_{3}\left(\mathfrak{M}_{(1)}\right)$ are consequences of those of $\mathfrak{M}_{(1)}$ plus an additional law $u$ with the property that $u^{2}$ is a law of $\mathfrak{M}_{(1)}$. Further she shows that if $U$ is the subgroup generated by the values of $u$ in $F_{\infty}\left(\mathfrak{M}_{(1)}\right)$, then $F_{\infty}\left(\mathfrak{M}_{(1)}\right) / U$ is isomorphic to the group $M_{3}$ of $3 \times 3$ matrices mentioned above. Thus it follows from (2) that $d\left(\operatorname{Var}\left(M_{3}\right)\right)=2$.

In Section 4 we show that not only is $d\left(\mathfrak{M}_{(2)}=4\right.$ (Theorem 4.6 ), but that

$$
\operatorname{Var} F_{2}\left(\mathfrak{M}_{(2)}\right)=\operatorname{Var} F_{3}(\mathfrak{M})_{(2)}<\operatorname{Var} F_{4}\left(\mathfrak{M}_{(2)}\right)=\mathfrak{M}_{(2)}
$$

which is the chain (2) with $\mathfrak{M}_{(1)}$ replaced by $\mathfrak{M}_{(2)}$. Our investigations, in Section 5 , regarding $\mathfrak{M}_{(c)}(c \geqq 3)$ are not so complete. However, while we have not determined the precise chain (1) for these cases, we are able to verify that for $c \geqq 3 \operatorname{Var} F_{k-1}\left(\mathfrak{M}_{(c)}\right)$ is properly contained in $\operatorname{Var} F_{k}\left(\mathfrak{M}_{(c)}\right)$ for $k=2, \cdots, c-1$. The proof uses methods similar to those in Levin [6].

Another problem for the varieties of the form $[\mathfrak{U}, \mathfrak{E}]$ is that of deciding when the centre of $F /[\mathrm{U}(F), F]$ is precisely $\mathrm{U}(F) /[\mathrm{U}(F), F]$, where $F$ is a free group of finite or countably infinite rank. This, for instance, is the case if $\mathfrak{U}=\mathfrak{N}_{c}$ (Witt, cf. [7] 31.63) or $\mathfrak{U}=\mathfrak{M}$ (follows from the fact that the centre of $F(\mathfrak{M})$ is trivial [1]). On the other hand, Cossey [3] has shown that this is not the case for $\mathfrak{U}=$ $\operatorname{Var} S L(2,5)$. As a by-product of our results, we show in Section 6 that the centre of $F /\left[F^{\prime \prime}, F, F\right]$ is precisely $\left[F^{\prime \prime}, F\right] /\left[F^{\prime \prime}, F, F\right]$.

We are thankful to Dr. M. F. Newman for his comments on an earlier draft of the paper.

\section{The Variety $\mathfrak{M}$}

Let $Z G$ be the integral group ring of a free abelian group $G$ freely generated by $x_{1}, x_{2}, \cdots$, and let $T_{2}=Z G\left[\Lambda_{2}\right]$ be the $Z G$-algebra in the set $\Lambda_{2}=\left\{\lambda_{21}^{(k)}\right.$; $k=1,2, \cdots\}$ of commuting indeterminates. Let $M_{2}$ be the multiplicative group of $2 \times 2$ matrices (over $T_{2}$ ) generated by 


$$
\left[\begin{array}{ll}
1 & 0 \\
\lambda_{21}^{(k)} & x_{k}
\end{array}\right]=X_{k}^{(2)},
$$

for $k=1,2, \cdots$. Let $F$ be the (absolutely) free group freely generated by $x_{1}, x_{2}, \cdots$ and let $\phi_{2}: F \rightarrow M_{2}$ be the homomorphism of $F$ onto $M_{2}$ defined by $\phi_{2}\left(x_{k}\right)=X_{k}^{(2)}$. Define a mapping $\alpha_{21}: F \rightarrow T_{2}$ by $\alpha_{21}(w)=21$-entry of the matrix $\phi_{2}(w)$ for all $w \in F$.

LemMA 2.1. (Magnus, $c f$. [7] 36.12). $\alpha_{21}(w)=0$ if and only if $w \in F^{\prime \prime}$. In particular $F / F^{\prime \prime}$ is isomorphic to $M_{2}$ under the natural mapping $x_{k} F^{\prime \prime} \rightarrow X_{k}^{(2)}$.

Since $G \cong F / F^{\prime}$, we may identify $G$ with $F / F^{\prime}$ (and $Z G$ with $Z\left(F / F^{\prime}\right)$ correspondingly) by identifying $x_{k}$ with $x_{k} F^{\prime}$. Thus if $w=w\left(x_{1}, \cdots, x_{n}\right) \in F^{\prime}$, then we may write

$$
w \equiv \prod_{n \geqq i>j \geqq 1}\left[x_{i}, x_{j}\right]^{a_{i j}}\left(\bmod F^{\prime \prime}\right),
$$

where $q_{i j}=q_{i j}\left(x_{1}, \cdots, x_{n}\right) \in Z G$.

For each $l, k \in\{1,2, \cdots\}$ and each $t \in Z$ we define an endomorphism $\theta_{l, k, t}$ of $F$ and an endomorphism $\bar{\theta}_{l, k, t}$ of $Z G$ as follows:

$$
\begin{aligned}
& \theta_{l, k, t}\left(x_{l}\right)=x_{k}^{t}, \theta_{l, k, t}\left(x_{i}\right)=x_{i} \text { for } i \neq l, \\
& \bar{\theta}_{l, k, t}\left(x_{l}\right)=x_{k}^{t}, \bar{\theta}_{l, k, t}\left(x_{i}\right)=x_{i} \text { for } i \neq l .
\end{aligned}
$$

LEMMA 2.2. If $w \in F^{\prime}$, then $\alpha_{21}(w)=\Sigma_{i} p_{i} \lambda_{21}^{(i)}$, where each $p_{i}$ is a uniquely determined element of $Z G$. Further if $\alpha_{21}\left(\theta_{l \cdot k, t}(w)\right)=\Sigma_{i} q_{i} \lambda_{21}^{(i)}$, then for all $i \notin\{l, k\}$, $q_{i}=\bar{\theta}_{l, k, t}\left(p_{i}\right)$. (Here $\theta, \bar{\theta}$ are as defined in $\left.(6),(7)\right)$.

Proof. It is clear that $\alpha_{21}(w)$ will be an expression of the form $\Sigma_{i} p_{i} \lambda_{21}^{(i)}$ and since $\lambda_{21}^{(i)}$ are linearly independent over $Z G, p_{i}$ are unique. Replacing $x_{k}$ by $x_{l}^{i}$ in $w$ has the effect of changing the corresponding matrix expression $\phi_{2}(w)$ by replacing $X_{k}^{(2)}$ by $\left(X_{l}^{(2)}\right)^{t}$. Thus if $i \notin\{k, l\}$, the coefficient of $\lambda_{21}^{(t)}$ in $\alpha_{21}\left(\theta_{l, k, t(w))}\right.$ is precisely $\bar{\theta}_{l, k, t}(p$,$) .$

For any $p \in Z G$ let $e_{k}(p)$ denote the maximum of the absolute values of the exponents of $\boldsymbol{x}_{k}$ occurring in $p$. The following lemma will have repeated applications in the sequel.

LEMMA 2.3. Let $p \in Z G, p \neq 0$. For any integers $l, k, \bar{\theta}_{l, k, t}(p) \neq 0$ whenever $|t| \geqq 2 e_{k}(p)+1$.

Proof. The lemma follows immediately from the observation that if $\left|s_{1}\right|<|s|$ $\left(s_{1} \neq s\right)$ and $s_{2} \geqq 2|s|+1$ then the equation $i s_{2}+s_{1}=j s_{2}+s$ has no integral solution, from which it follows that if $s_{2} \geqq 2|s|+1$, then $x_{l}^{l} x_{k}^{s_{1}}-x_{l}^{j} x_{k}^{s}$ will not vanish for any replacement of $\boldsymbol{x}_{l}$ by $\boldsymbol{x}_{k}^{s_{2}}$.

We conclude this section by giving an alternate proof of the following result. 
THEOREM 2.4. (cf. [2]). For $n \geqq 2 F_{n}(\mathfrak{M})$ is residually $F_{2}(\mathfrak{M})$.

Proof. It is enough to show that for $n \geqq 3, F_{n}(\mathfrak{M})$ is residually $F_{n-1}(\mathfrak{M})$. Let $w=w\left(x_{1}, \cdots, x_{n}\right)$ be an element of $F \backslash F^{\prime \prime}$. If $w \notin F^{\prime}$ then $\theta_{i, i, 0}(w) \notin F^{\prime}$ for some $i \in\{1, \cdots, n\}$. Thus we may assume that $w \in F^{\prime} \mid F^{\prime \prime}$. By Lemmas 2.2 and $2.1, \alpha_{21}(w)$ $=p_{1} \lambda_{21}^{(1)}+\cdots+p_{n} \lambda_{21}^{(n)} \neq 0$, and we may assume, without loss of generality, that $p_{1}=p_{1}\left(x_{1}, \cdots, x_{n}\right) \neq 0$. Since $n \geqq 3$, by Lemma 2.2 the coefficient of $\lambda_{21}$ in the expansion of $\alpha_{21}\left(\theta_{n, n-1, t}(w)\right)$ is precisely $\theta_{n, n-1, t}\left(p_{1}\right)$ which by Lemma 2.3 is non-zero for a large enough $t$. It follows by Lemma 2.1 that $\theta_{n, n-1,1}(w) \notin F^{\prime \prime}$. This completes the proof of the theorem.

3. The variety $\mathfrak{M}_{(1)}(=[\mathfrak{M}, \mathfrak{E}])$

As in Section 2 let $\Lambda_{3}=\left\{\lambda_{i, i-1}^{(k)} ; i=2,3 ; k=1,2, \cdots\right\}$ and let $T_{3}=Z G\left[\Lambda_{3}\right]$. Let $M_{3}$ be the group of $3 \times 3$ matrices (over $T_{3}$ ) generated by

$$
\left[\begin{array}{lll}
1 & 0 & 0 \\
\lambda_{21}^{(k)} & x_{k} & 0 \\
0 & \lambda_{32}^{(k)} & 1
\end{array}\right]=X_{k}^{(3)},
$$

for $k=1,2, \cdots$ and let $\phi_{3}$ be the homomorphism of $F$ onto $M_{3}$ defined by $\phi_{3}\left(x_{k}\right)$ $=X_{k}^{(3)}$ for $k=1,2, \cdots$. Further let $\alpha_{i j}(3 \geqq i>j \geqq 1)$ be the mapping of $F$ into $T_{3}$ defined by $\alpha_{i j}(w)=i j$-entry of the matrix $\phi_{3}(w)$ for all $w \in F$.

LemMa 3.1. (Gupta [4]). Let $w \in F^{\prime \prime}$. Then $\alpha_{31}(w)=0$ if and only if $w=w_{1} w_{2}$, where $w_{1}$ is a product of values of the word

$$
\begin{aligned}
u_{1234}(x)= & {\left[x_{1}^{-1}, x_{2}^{-1} ; x_{3}, x_{4}\right]\left[x_{1}^{-1}, x_{3}^{-1} ; x_{4}, x_{2}\right]\left[x_{1}^{-1}, x_{4}^{-1} ; x_{2}, x_{3}\right] } \\
& {\left[x_{3}^{-1}, x_{4}^{-1} ; x_{1}, x_{2}\right]\left[x_{4}^{-1}, x_{2}^{-1} ; x_{1}, x_{3}\right]\left[x_{2}^{-1}, x_{3}^{-1}, x_{1}, x_{4}\right], }
\end{aligned}
$$

and $w_{2} \in\left[F^{\prime \prime}, F\right]$.

LEMMA 3.2. (Gupta [4]). $F_{3}\left(\mathfrak{M}_{(1)}\right)$ is isomorphic to the subgroup of $M_{3}$ generated by $X_{1}^{(3)}, X_{2}^{(3)}, X_{3}^{(3)}$.

LEMmA 3.3. (Gupta [5]). $u_{1234}(x) \notin\left[F^{\prime \prime}, F\right]$ but $u_{1234}^{2}(x) \in\left[F^{\prime \prime}, F\right]$, where $u_{1234}(x)$ is defined by (9). Further if $w=w\left(x_{1}, \cdots, x_{n}\right)(n \geqq 4)$ is an n-variable word in $F^{\prime \prime}$ such that $\alpha_{31}(w)=0$, then

$$
w=\prod_{1 \leqq i<j<k<1 \leqq n} u_{i j k l}^{\varepsilon(i j k l)}(x)\left(\bmod \left[F^{\prime \prime}, F\right]\right),
$$

where $u_{i j k l}(x)$ is defined as in $(9)$ and $\varepsilon(i j k l) \in\{0,1\}$.

The next lemma is analogous to Lemma 2.2 and the proof is essentially the same.

Lemma 3.4. If $w \in F^{\prime \prime}$, then $\alpha_{31}(w)=\Sigma_{i, j} p_{i j} \lambda_{32}^{(i)} \lambda_{21}^{(j)}$, where each $p_{i j}$ is a uniquely 
determined element of ZG. Further if $\alpha_{31}\left(\theta_{l, k, t}(w)\right)=\Sigma_{i, j} q_{i j} \lambda_{32}^{(i)} \lambda_{21}^{(j)}$, then for all $i, j \notin\{l, k\}, q_{i j}=\bar{\theta}_{l, k, t}\left(p_{i j}\right)$, where $\theta, \bar{\theta}$ are defined in (6), (7).

LEMMA 3.5. Let $w=w\left(x_{1}, \cdots, x_{n}\right)(n \geqq 2) \in F^{\prime \prime}$ be an $n$-variable word such that $\alpha_{31}(w) \neq 0$. Then there is an automorphism $\xi$ of $F$ such that for some $i \in\{1, \cdots, n\}$ the coefficient of $\lambda_{32}^{(i)} \lambda_{21}^{(i)}$ in the expansion of $\alpha_{31}(\xi(w))$ is non-zero.

Proof. Let $\alpha_{31}(w)=\Sigma_{k, l} p_{k} \lambda_{32}^{(k)} \lambda_{21}^{(l)}$. If for some $i, p_{i i} \neq 0$ then we take $\xi$ to be the identity automorphism of $F$. Otherwise, we may assume that for some $i$, $j \in\{1, \cdots, n\}(i \neq j), p_{i i}=0=p_{j j}$ and one of $p_{i j}, p_{j i}$ is non-zero. Let $\xi_{1}$ be the automorphism of $F$ which maps $x_{j}$ to $x_{l} x_{j}$ and $x_{k}$ to $x_{k}$ for $k \neq j$, and $\xi_{2}$ be the corresponding automorphism mapping $x_{j}$ to $x_{j} x_{i}$ and $x_{k}$ to $x_{k}$ for $k \neq j$. Let $\alpha_{31}\left(\xi_{1}(w)\right)=\Sigma_{k, 1} q_{k l} \lambda_{32}^{(k)} \lambda_{21}^{(l)}$ and $\alpha_{31}\left(\xi_{2}(w)\right)=\Sigma_{k, l} r_{k l} \lambda_{32}^{(k)} \lambda_{21}^{(l)}$. One verifies that

$$
q_{i i}=\bar{p}_{i j}+\boldsymbol{x}_{j} \bar{p}_{j i} \text { and } r_{i i}=\boldsymbol{x}_{j} \bar{p}_{i j}+\bar{p}_{j i}
$$

where $\bar{p}$ is obtained from $p$ on replacing $x_{j}$ by $x_{j} x_{i}$. If both $q_{i i}$ and $r_{i i}$ are zero then both $\bar{p}_{i j}$ and $\bar{p}_{j i}$ must be zero and equivalently both $p_{i j}$ and $p_{j l}$ must be zero, contrary to the assumption.

We can now prove,

THEOREM 3.6. Let $\mathfrak{G}=\operatorname{Var}\left(M_{3}\right)$. Then for all $n \geqq 2, F_{n}(\mathfrak{G})$ is residually $F_{2}(\mathfrak{G})$. In particular $F_{3}\left(\mathfrak{M}_{(1)}\right)$ is residually $F_{2}\left(\mathfrak{M}_{(1)}\right)$.

Proof. Let $w=w\left(x_{1}, \cdots, x_{n}\right)(n \geqq 3)$ be an $n$-variable word in $F$ such that $w \notin \mathscr{G}(F)<F^{\prime \prime}$. If $w \notin F^{\prime \prime}$ then, by Theorem $2.4, \theta_{l, k, l}(w) \notin F^{\prime \prime}$ for some $l, k \in\{1, \cdots, n\}$ $(l \neq k)$ and some $t \in Z$. Thus we may assume that $w \in F^{\prime \prime}$. Using an automorphism $\xi$ of $F$, if necessary, we may, by Lemma 3.5, assume that the coefficient $p_{i i}$ of $\lambda_{32}^{(i)} \lambda_{21}^{(i)}$ in the expansion of $\alpha_{31}(w)$ is non-zero for some $i \in\{1, \cdots, n\}$. It follows, by Lemma 2.3, that $\bar{\theta}_{l, k, t}\left(p_{i i}\right) \neq 0$ for $i \notin\{l, k\}(l \neq k)$. Thus by Lemma 3.4, $\theta_{l, k, t}$ $(w) \notin \mathfrak{G}(F)$. The second part of the theorem uses Lemma 3.2.

We conclude this section by proving the following result.

THEOREM 3.7. For $n \geqq 4, F_{n}\left(\mathfrak{M}_{(1)}\right)$ is residually $F_{4}\left(\mathfrak{D}_{(1)}\right)$.

Proof. Let $w=w\left(x_{1}, \cdots, x_{n}\right)(n \geqq 5)$ be an $n$-variable word in $F \backslash\left[F^{\prime \prime}, F\right]$. As in Theorem 3.6 we may assume that $w \in F^{\prime \prime} \mid\left[F^{\prime \prime}, F\right]$. Further, if $\alpha_{31}(w) \neq 0$ then, as in Theorem 3.6, $\theta_{l, k \cdot t}(\xi(w)) \notin\left[F^{\prime \prime}, F\right]$. Thus we may assume that $\alpha_{31}(w)=0$ so that, by Lemma 3.3,

$$
w=\prod_{1 \leqq i<j<k<l \leqq n} u_{i j k l}^{\varepsilon(i j k l)}\left(\bmod \left[F^{\prime \prime}, F\right]\right)
$$

and for some $i<j<k<l, \varepsilon(i j k l) \neq 0$. Since $n \geqq 5$, we can choose $r \notin\{i, j, k, l\}$. By Lemma 3.3, $\theta_{r, r, 0}(w) \notin\left[F^{\prime \prime}, F\right]$. This completes the proof of the theorem. 


\section{The variety $\mathfrak{M}_{(2)}(=[\mathfrak{M}, \mathfrak{E}, \mathfrak{E}])$}

In this section we first of all show that $d\left(\mathfrak{M}_{(2)}\right)>3$. We do this by exhibiting a 4-variable word which is not a law in $F_{4}\left(\mathfrak{M}_{(2)}\right)$ but is a law in $F_{3}\left(\mathfrak{M}_{(2)}\right)$.

THEOREM 4.1. Let $w=\left[u_{1234}(x), x_{4}\right]$, where $u_{1234}(x)$ is defined by (10). Then $w$ is a law in $F_{3}\left(\mathfrak{M}_{(2)}\right)$ but not a law in $F_{4}\left(\mathfrak{M}_{(2)}\right)$. In particular $\operatorname{Var} F_{3}\left(\mathfrak{M}_{(2)}\right)$ $<\operatorname{Var} F_{4}\left(\mathfrak{M}_{(2)}\right)$.

ProOF. Since $u_{1234}(x)$ is a law in $F_{3}\left(\mathfrak{M}_{(1)}\right)$ (Gupta [4]), it follows that $w$ is a law in $F_{3}\left(\mathfrak{M}_{(2)}\right)$. To complete the proof it suffices to show that $w$ is not a law in $F_{4}\left(\mathfrak{M}_{(2)} \wedge \mathfrak{N}_{7}\right)$.

Expanding $w$ modulo $\gamma_{8}(F)\left[F^{\prime \prime}, F, F\right]$ shows that

$$
\begin{aligned}
w= & {\left[x_{1}, x_{2} ; x_{1}, x_{2}, x_{3}, x_{4} ; x_{4}\right]\left[x_{1}, x_{3} ; x_{1}, x_{3}, x_{2}, x_{4} ; x_{4}\right]\left[x_{1}, x_{4} ; x_{1}, x_{4}, x_{2}, x_{3} ; x_{4}\right] } \\
& {\left[x_{2}, x_{3} ; x_{2}, x_{3}, x_{1}, x_{4} ; x_{4}\right]\left[x_{2}, x_{4} ; x_{2}, x_{4}, x_{1}, x_{3} ; x_{4}\right]\left[x_{3}, x_{4} ; x_{3}, x_{4}, x_{1}, x_{2} ; x_{4}\right] }
\end{aligned}
$$

(cf. [5]). Since the frequency of generators is diferent in each factor, as words in $\gamma_{7}(F)$ the factors of $w$ are independent of each other modulo $\gamma_{8}(F)$. However, it is readily verified that modulo $\gamma_{8}(F),\left[F^{\prime \prime}, F, F\right]$ is generated by all commutators of the form $\left[x_{i 1}, x_{i 2} ; x_{i 3}, x_{i 4} ; x_{i 5} ; x_{i 6}\right]$ plus those of the forms $\left[x_{i 1}, x_{i 2} ; x_{i 3}, x_{i 4} ; x_{i 5}\right.$; $\left.x_{i 6} ; x_{i 7}\right],\left[x_{i 1}, x_{i 2} ; x_{i 3}, x_{i 4} ; x_{i 5}, x_{i 6} ; x_{i 7}\right],\left[x_{i 1}, x_{i 2}, x_{i 3} ; x_{i 4}, x_{i 5} ; x_{i 6} ; x_{i 7}\right]$ and $\left[x_{i 1}, x_{i 2}\right.$; $\left.x_{i 3}, x_{i 4} ; x_{i 5} ; x_{i 6}, x_{i 7}\right]$ where $i 1, i 2, \cdots, i 7 \in\{1,2, \cdots\}$. In particular if $w \equiv 1$ (modulo $\left.\gamma_{8}(F)\left[F^{\prime \prime}, F, F\right]\right)$ then it is not difficult to verify that in fact $w \in\left[\gamma_{3}(F), \gamma_{2}(F), F, F\right]$ $\gamma_{8}(F)$. Since the generators of weight 7 cannot alter the frequency pattern of any factor of $w$, it follows that if $w$ lies in $\gamma_{8}(F)\left[F^{\prime \prime}, F, F\right]$ then each factor of $w$ lies in $\gamma_{8}(F)\left[F^{\prime \prime}, F, F\right]$, and in particular, $\left[x_{1}, x_{2} ; x_{1}, x_{2}, x_{1}, x_{2} ; x_{2}\right] \in \gamma_{8}(F)\left[F^{\prime \prime}, F, F\right]$. In what follows we shall show that $\left[x_{1}, x_{2} ; x_{1}, x_{2}, x_{1}, x_{2} ; x_{2}\right]$ is in fact non-trivial modulo $\gamma_{8}(F)\left[F^{\prime \prime}, F, F\right]$.

Let $H$ be the free group of class 7 freely generated by $a, b$ and let $N_{1}$ be the normal subgroup of $H$ generated by all basic commutators ([7],31.51) of weight 7 other than the following three commutators:

$c_{1}=[b, a, a, b, b ; b, a], \quad c_{2}=[b, a, a, b ; b, a, b]$ and $c_{3}=[b, a, b, b ; b, a, a]$.

Let $N_{2}$ be the normal subgroup of $H$ generated by $N_{1}, c_{1}^{2}, c_{2}^{2}, c_{3}^{2}, c_{1} c_{3}^{-1}$. Then

$$
\begin{aligned}
d= & {[b, a, a, b ; b, a ; b]=[b, a, a, b, b ; b, a][b, a, a, b ; b, a, b] \text { by the Witt } } \\
& \text { identity }([7], 33.34) \\
= & c_{1} c_{2} \notin N_{2} .
\end{aligned}
$$

We next observe that modulo $N_{2}$,

$$
\begin{aligned}
& {[b, a, b ; b, a ; b ; a] \equiv[b, a, b ; b, a ; a ; b] \equiv[b, a, b, a ; b, a ; b][b, a, b ; b, a, a ; b]} \\
& \equiv d c_{3} c_{2}^{-1}=c_{1} c_{2} c_{3} c_{2}^{-1}=c_{1} c_{3} \equiv 1, \text { and }[b, a, a ; b, a ; b ; b] \\
& \equiv[b, a, a, b ; b, a ; b][b, a, a ; b, a, b ; b] \equiv d c_{2} c_{3}^{-1}=c_{1} c_{2} c_{2} c_{3}^{-1}=c_{1} c_{3}^{-1} \equiv 1
\end{aligned}
$$


Thus $\mathrm{H} / \mathrm{N}_{2}$ is a centre-by-centre-by-metabelian group of class 7 in which $d=[b, a, a, b ; b, a ; b]$ is non-trivial. This completes the proof of the theorem. We now construct an $\mathfrak{M}_{(2)}$-group which will be useful in the sequel.

As in Sections 2 and 3 let $\Lambda_{4}=\left\{\lambda_{t, i-1}^{(k)} ; i=2,3,4 ; k=1,2, \cdots\right\}$ and $T_{4}$ $=Z G\left[\Lambda_{4}\right]$. Let $M_{4}$ be the group of $4 \times 4$ matrices (over $T_{4}$ ) generated by

$$
\left[\begin{array}{llll}
1 & 0 & 0 & 0 \\
\lambda_{21}^{(k)} & \boldsymbol{x}_{k} & 0 & 0 \\
0 & \lambda_{32}^{(k)} & 1 & 0 \\
0 & 0 & \lambda_{43}^{(k)} & 1
\end{array}\right]=X_{k}^{(4)}
$$

for $k=1,2, \cdots$. Let $\phi_{4}$ be the homomorphism of $F$ onto $M_{4}$ defined by $\phi_{4}\left(x_{k}\right)$ $=X_{k}^{(4)}$ for $k=1,2, \cdots$ and let $\alpha_{i j}(4 \geqq i>j \geqq 1)$ be the mapping of $F$ into $T_{4}$ defined by $\alpha_{i j}(w)=i j$-entry of the matrix $\phi_{4}(w)$ for all $w \in F$. Using matrix multiplication the following lemma is routinely verified.

LEMMA 4.2. (i) If $w \in\left[F^{\prime \prime}, F, F\right]$, then $w \in$ kernel of $\phi_{4}$;

(ii) $\left[u_{1234}(x), x_{5}\right] \in$ kernel of $\phi_{4}$;

(iii) If $w \in F^{\prime \prime}$, then $\alpha_{41}\left[w, x_{k}\right]=-\lambda_{43}^{(k)} x_{31}(w)$.

We now establish the following useful analogue of Lemma 3.5.

Lemma 4.3. Let $w=w\left(x_{1}, \cdots, x_{n}\right)(n \geqq 2)$ be an $n$-variable word in $\left[F^{\prime \prime}, F\right]$ such that $\alpha_{41}(w) \neq 0$. Then there is an automorphism $\xi$ of $F$ such that for some $i \in\{1, \cdots, n\}$, the coefficient of $\lambda_{43}^{(i)} \lambda_{32}^{(i)} \lambda_{21}^{(i)}$ is non-zero in the expansion of $\left.\alpha_{41} \xi(w)\right)$.

Proof. By Lemmas 4.2 and 3.4 we write

$$
\alpha_{41}(w)=\sum_{i, j, k} p_{i j k} \lambda_{43}^{(i)} \lambda_{32}^{(j)} \lambda_{21}^{(k)}
$$

where $p_{i j k}$ are uniquely determined elements of $Z G$ and for some $i, j, k \in\{1, \cdots, n\}$, $p_{i j k} \neq 0$. Let $i, j \in\{1, \cdots, n\}(i \neq j)$ be fixed and let $\xi_{1}, \xi_{2}, \xi_{3}$ be automorphisms of $F$ defined as follows: $\xi_{1}\left(x_{j}\right)=x_{i} x_{j}, \xi_{1}\left(x_{k}\right)=x_{k}$ for $k \neq j ; \xi_{2}\left(x_{j}\right)=x_{j} x_{i}, \xi_{2}\left(x_{k}\right)$ $=x_{k}$ for $k \neq j ; \xi_{3}\left(x_{i}\right)=x_{i}^{-1}, \xi_{3}\left(x_{k}\right)=x_{k}$ for $k \neq i$. Let

$$
\begin{aligned}
& \alpha_{41}\left(\xi_{1}(w)\right)=\sum_{i, j, k} q_{i j k} \lambda_{43}^{(i)} \lambda_{32}^{(j)} \lambda_{21}^{(k)} ; \alpha_{41}\left(\xi_{2}(w)\right)=\sum_{i, j, k} r_{i j k} \lambda_{43}^{(i)} \lambda_{32}^{(j)} \lambda_{21}^{(k)} ; \text { and } \\
& \alpha_{41}\left(\xi_{3}(w)\right)=\sum_{i, j, k} s_{i j k} \lambda_{43}^{(i)} \lambda_{32}^{(j)} \lambda_{21}^{(k)} .
\end{aligned}
$$

For $p \in Z G$, let $p^{*}$ be the element of $Z G$ obtained from $p$ on replacing $x_{i}$ by $\boldsymbol{x}_{i}^{-1}$ and $\vec{p}$ be the element of $Z G$ obtained from $p$ on replacing $x_{j}$ by $x_{j} x_{i}$. If $p_{i i i}=p_{j j j}$ $=0$, then using matrix multiplication the following can be verified:

$$
q_{i i i}=\bar{p}_{i i j}+x_{j} \bar{p}_{i j i}+\bar{p}_{j i i}+x_{j} \bar{p}_{j j i}+\bar{p}_{j i j}+x_{j} \bar{p}_{i j j}
$$




$$
\begin{aligned}
& r_{i i i}=x_{j} \bar{p}_{i i j}+\bar{p}_{i j i}+\bar{p}_{j i i}+\bar{p}_{j j i}+x_{j} \bar{p}_{j i j}+x_{j} \bar{p}_{i j j} ; \\
& s_{i i j}=x_{i}^{-1} p_{i i j}^{*}, s_{i j i}=x_{i}^{-1} p_{i j i}^{*}, s_{j j i}=-x_{i}^{-1} p_{j j i}^{*} \text { and } s_{j i j}=-x_{i}^{-1} p_{j i j}^{*} \\
& q_{j j i}=\bar{p}_{j j i}, q_{j i j}=x_{i} \tilde{p}_{j i j} \text { and } q_{i j i}=\bar{p}_{i j i}+\bar{p}_{j j i}+\bar{p}_{i j j} \\
& q_{k i l}=\bar{p}_{k i j}+x_{j} \bar{p}_{k j i}+\bar{p}_{k i i}+x_{j} \bar{p}_{k j j} ; \text { and } \\
& r_{k i i}=x_{j} \bar{p}_{k i j}+\bar{p}_{k j i}+\bar{p}_{k i i}+x_{j} \bar{p}_{k j j} .
\end{aligned}
$$

To complete the proof of the lemma, let us assume that,

$$
p_{i i i}=p_{j j j}=q_{i i i}=q_{j j j}=r_{i i i}=r_{j j j}=s_{i i i}=s_{j j j}=0 .
$$

Then from (13) and (14) we conclude that

$$
p_{i i j}-p_{i j i}=p_{j j i}-p_{j i j} \text {, and hence also } s_{i j j}-s_{i j i}=s_{j j i}-s_{j i j} \text {. }
$$

Using (15), this last equation yields

$$
p_{i i j}-p_{i j i}=-p_{j j i}+p_{j i j}
$$

which together with the first equation in (20) gives

$$
p_{i i j}=p_{i j i} \text { and } p_{j j i}=p_{j i j} \text {, and hence also } q_{i j j}=q_{i j i} \text { and } q_{j j i}=q_{j i j} \text {. }
$$

Using (16) the last equation in (21) together with the second equation in (21) gives $p_{j j i}=0=p_{j i j}$; by symmetry,

(22) $p_{i i j}=p_{i j i}=p_{j j i}=p_{j i j}=0$, and similarly $q_{i i j}=q_{i j i}=q_{j j i}=q_{j i j}=0$.

Using (22) in the last equation in (16) yields

$$
p_{i j j}=0 \text { and (by symmetry) } p_{j i i}=0 .
$$

Thus we have shown that if (19) holds for any $i, j \in\{1, \cdots, n\}$, then

$$
p_{i i j}=p_{i j l}=p_{j i i}=p_{j j i}=p_{j i j}=p_{i j j}=0,
$$

and the same for the corresponding $q, r, s$ terms. Assuming (19) for every pair $i, j \in\{1, \cdots, n\}(i \neq j)$, if $k \notin\{i, j\}$, then $q_{k i i}=r_{k i i}=\bar{p}_{k i i}=\bar{p}_{k j j}=0$, so that from (17) and (18) we get as in the proof of Lemma 3.5, $p_{k i j}=0$, which implies by (11) that $\alpha_{41}(w)=0$, contrary to the hypothesis. This completes the proof of the lemma.

As an immediate consequence of Lemma 4.3, we prove the following.

THEOREM 4.4. $F_{3}\left(\mathfrak{M}_{(2)}\right)$ is residually $F_{2}\left(\mathfrak{M}_{(2)}\right)$.

Proof. Let $w=w\left(x_{1}, x_{2}, x_{3}\right) \in F \backslash\left[F^{\prime \prime}, F, F\right]$. By Theorem 3.6, we may assume that $w \in\left[F^{\prime \prime}, F\right] \backslash\left[F^{\prime \prime}, F, F\right]$. If $\alpha_{41}(w) \neq 0$ then, as in the proof of Theorem 3.6, using Lemma 4.3 we can map $w$ to a 2-variable word which does not belong to $\left[F^{\prime \prime}, F, F\right]$. If $\alpha_{41}(w)=0$, then we may write $w \equiv\left[v_{1}, x_{1}\right]\left[v_{2}, x_{2}\right]\left[v_{3}, x_{3}\right]$ 
$\bmod \left[F^{\prime \prime}, F, F\right]$ ), where $v_{1}, v_{2}, v_{3} \in F^{\prime \prime}$ and $\alpha_{31}\left(v_{i}\right)=0$ for $i=1,2,3$ (by Lemma 4.2 (iii)). By Lemma 3.2, each $v_{i} \in\left[F^{\prime \prime}, F\right]$ and hence $w \in\left[F^{\prime \prime}, F, F\right]$, contrary to the assumption.

For the proof of our final result in this section, we need the following lemma.

LEMMA 4.5. Let

$$
\begin{aligned}
w= & {\left[u_{2345}(x), x_{1}\right]\left[u_{1345}(x), x_{2}\right] } \\
& {\left[u_{1245}(x), x_{3}\right]\left[u_{1235}(x), x_{4}\right]\left[u_{1234}(x), x_{5}\right], }
\end{aligned}
$$

where $u_{i j k l}(x)$ is defined by (9). Then $w \in\left[F^{\prime \prime}, F, F\right]$.

Proof. If $v=\left[x_{1}^{-1}, x_{2}^{-1} ; x_{3}, x_{4}, x_{5}\right]\left[x_{1}^{-1}, x_{3}^{-1} ; x_{4}, x_{2}, x_{5}\right]\left[x_{1}^{-1}, x_{4}^{-1} ; x_{2}, x_{3}, x_{5}\right]$

$$
\left[x_{3}^{-1}, x_{4}^{-1} ; x_{1}, x_{2}, x_{5}\right]\left[x_{4}^{-1}, x_{2}^{-1} ; x_{1}, x_{3}, x_{5}\right]\left[x_{2}^{-1}, x_{3}^{-1} ; x_{1}, x_{4}, x_{5}\right]
$$

then working modulo $\left[F^{\prime \prime}, F\right]$, it can be verified directly that

$$
v \equiv 1 \text {. }
$$

Further, using the Witt identity

$\left[a, b, c^{a}\right]\left[c, a, b^{c}\right]\left[b, c, a^{b}\right]=1$ with $a=\left[x_{1}^{-1}, x_{2}^{-1}\right], b=\left[x_{3}, x_{4}\right], c=x_{5}$ and working modulo $\left[F^{\prime \prime}, F, F\right]$ gives $\left[x_{1}^{-1}, x_{2}^{-1} ; x_{3}, x_{4} ; x_{5}\right]\left[x_{5},\left[x_{1}^{-1}, x_{2}^{-1}\right],\left[x_{3}, x_{4}\right]^{x_{5}}\right]$ $\left[x_{3}, x_{4}, x_{5},\left[x_{1}^{-1}, x_{2}^{-1}\right]\right] \equiv 1$ and hence

$$
\left[x_{1}^{-1}, x_{2}^{-1} ; x_{3}, x_{4} ; x_{5}\right]\left[x_{1}^{-1}, x_{2}^{-1}, x_{5}^{-1} ; x_{3}, x_{4}\right]^{x_{4}}\left[x_{1}^{-1}, x_{2}^{-1} ; x_{3}, x_{4}, x_{5}\right]^{-1} \equiv 1 .
$$

To complete the proof of the lemma, we first expand $w$ applying (26) to each factor. Next we note, using (25), that the 6-weight contributions of each $\left[u_{i j k l}(x), x_{t}\right]$ lie in $\left[F^{\prime \prime}, F, F\right]$. Finally, the remaining 5-weight commutators in $w$ can be rearranged to form a product of elements of the form

$$
\begin{gathered}
{\left[\left[x_{i}^{-1}, x_{j}^{-1}\right],\left[x_{k}, x_{l}, x_{m}\right]\left[x_{l}, x_{m}, x_{k}\right]\left[x_{m}, x_{k}, x_{l}\right]\right] \text { and }} \\
{\left[\left[x_{i}^{-1}, x_{j}^{-1}, x_{k}^{-1}\right]\left[x_{j}^{-1}, x_{k}^{-1}, x_{i}^{-1}\right]\left[x_{k}^{-1}, x_{i}^{-1}, x_{j}^{-1}\right],\left[x_{l}, x_{m}\right]\right]^{-1}}
\end{gathered}
$$

which belong to $\left[F^{\prime \prime}, F, F\right]$.

We are now in a position to prove the following theorem.

THEOREM 4.6. For each $n \geqq 4, F_{n}\left(\mathfrak{M}_{(2)}\right)$ is residually $F_{4}\left(\mathfrak{M}_{(2)}\right)$.

Proof. Let $w=w\left(x_{1}, \cdots, x_{n}\right)(n \geqq 5)$ be an $n$-variable word in $F \backslash\left[F^{\prime \prime}, F, F\right]$. Then as in Theorem 3.6, we may assume that $w \in\left[F^{\prime \prime}, F\right]$, so that $w \equiv \Pi_{i=1}^{n}\left[v_{i}, x_{i}\right]$ $\left(\bmod \left[F^{\prime \prime}, F, F\right]\right)$, where $v_{i} \in F^{\prime \prime}$. By Lemma 4.2 (iii), $\alpha_{41}(w)=\sum_{i=1}^{n}-\lambda_{43}^{(i)} \alpha_{31}\left(v_{i}\right)$. There are two cases to be considered.

CASE I. $\left(\alpha_{41}(w) \neq 0\right)$. In this case, as in the proof of Theorem 4.4 , we can use Lemma 4.3 to map $w$ to an $(n-1)$-variable non-trivial word $\left(\bmod \left[F^{\prime \prime}, F, F\right]\right)$. 
CASE II. $\left(\alpha_{41}(w)=0\right)$. In this case $\alpha_{31}\left(v_{i}\right)=0$ for each $i=1, \cdots, n$. Thus by Lemma 3.3 each $v_{i}$ is of the form (10). If $n>5$ then $\theta_{k, k, 0}(w) \notin\left[F^{\prime \prime}, F, F\right]$ for some $k$ (by Theorem 4.1, $\left.\left[u_{1234}(x), x_{5}\right] \notin\left[F^{\prime \prime}, F, F\right]\right)$. If $n=5$, then $w \equiv\left[u_{2345}(x), x_{1}\right]^{\beta_{1}}$ $\cdots\left[u_{1234}(x), x_{5}\right]^{\beta_{5}}\left(\bmod \left[F^{\prime \prime}, F, F\right]\right)$, where $\beta_{1}, \cdots, \beta_{5} \in\{0,1\}$, by Lemma 3.3. Since $w \notin\left[F^{\prime \prime}, F, F\right]$, by Lemma $4.5 \beta_{i}=0$ and $\beta_{j}=1$ for some $i, j$, and we may assume, without loss of generality, that $\beta_{1}=0$ and $\beta_{2}=1$. Then $\theta_{12,1}(w)$ $=\left[u_{2345}(x), x_{2}\right] \notin\left[F^{\prime \prime}, F, F\right]$. This completes the proof of the theorem.

\section{The variety $\mathfrak{M}_{(c)}(c \geqq 3)$}

While we are unable to determine the precise chain (1) for $c \geqq 3$, our main result in this section goes some way towards the solution of this problem. Our method is similar to the one used in Levin [6].

Let $Z\left[y_{1}, \cdots, y_{m}\right](m \geqq 3)$ be the free associative $Z$-algebra in non-commuting indeterminates $y_{1}, \cdots, y_{m}$ and let $I_{m+5}$ be the ideal generated by all monomials of length $m+5$. Put $R=Z\left[y_{1}, \cdots, y_{m}\right] / I_{m+5}$. We first prove the following lemma.

Lemma 5.1. Let $\rho_{m}=\Sigma_{\sigma}|\sigma|\left\langle y, y_{1 \sigma}, \cdots, y_{m \sigma}\right\rangle$, where $y=\left\langle\left\langle y_{1}, y_{2}\right\rangle,\left\langle y_{3}, y_{4}\right\rangle\right\rangle$ and $\sigma$ runs through all permutation of $\{1,2, \cdots, m\}$ with $|\sigma|=1$ or -1 according as $\sigma$ is even or odd. Then $\rho_{m} \notin I_{m+5}$. (Here $\left\langle r_{1}, r_{2}\right\rangle$ denotes the Lie commutator $r_{1} r_{2}-r_{2} r_{1}$.)

Proof. Case I. ( $m$ odd).

If $\rho_{m} \equiv 0\left(\bmod \mathrm{I}_{m+5}\right)$, then the sum of the terms with left factor $y_{1}^{2}$ in the expansion of $\rho_{m}$ is in $I_{m+5}$. However, these occur precisely in the terms with left factor $y_{1} y$ and a straight-forward computation shows that this sum is $-y_{1} y \Sigma_{\sigma^{\prime}}\left|\sigma^{\prime}\right| y_{2 \sigma^{\prime}}$ $\cdots y_{m \sigma^{\prime}}$ (since $m$ is odd), where $\sigma^{\prime}$ runs through all permutations of $\{2, \cdots, m\}$, and this is clearly non-zero modulo $I_{m+5}$.

CASE II. ( $m$ even).

In this case we proceed as in Case I, but this time we consider terms with left factors $\left\langle y_{1}, y_{2}\right\rangle y$ and show that this sum is not in $\mathbf{I}_{m+5}$. The computation in this case is simplified by making use of the identity

$$
\left\langle y, \cdots, y_{i}, y_{j}, \cdots\right\rangle-\left\langle y, \cdots, y_{j}, y_{i}, \cdots\right\rangle=\left\langle y, \cdots,\left\langle y_{i}, y_{j}\right\rangle, \cdots\right\rangle
$$

to rewrite $\rho_{m}$ as

$$
\rho_{m}=\Sigma_{\mu}\left\langle y,\left\langle y_{1 .,}, y_{2 . .}\right\rangle, \cdots,\left\langle y_{(m-1) .}, y_{m . .}\right\rangle\right\rangle,
$$

where $\mu$ runs through all even permutations of $\{1, \cdots, m\}$ satisfying $(2 i-1) \mu<(2 i) \mu$ for $i=1, \cdots, m / 2$. We omit the rest of the details.

THEOREM 5.2. Var $F_{2}\left(\mathfrak{M}_{(c)}\right)<\cdots<\operatorname{Var} F_{c-1}\left(\mathfrak{M}_{(c)}\right)(c \geqq 4)$.

Proof. To show that Var $F_{c-2}\left(\mathfrak{M}_{(c)}\right)<\operatorname{Var} F_{c-1}\left(\mathfrak{M}_{(c)}\right)$, we consider the word 


$$
w_{c-1}=\Pi_{\sigma}\left[x, x_{1, s}, \cdots, x_{(c-1) \sigma}\right]^{|\sigma|}
$$

where $x=\left[x_{1}, x_{2} ; x_{1}, x_{3}\right]$ and $\sigma$ runs through all permutations of $\{1, \cdots, c-1\}$. It is immediate that $w_{c-1}$ is a law in $F_{c-2}\left(\mathfrak{M}_{(c)}\right)$. With $m=c-1$, the group $A(R)$ of units of $R$ belongs to $\operatorname{Var} F_{c-1}\left(\mathfrak{M}_{(c)}\right)$. Thus to see that $w_{c-1}$ is not a law in $F_{c-1}\left(\mathfrak{M}_{(c)}\right)$, we note by Lemma 5.1 that $\rho_{c-1} \notin I_{c+4}$. Finally to see that Var $F_{k-2}$ $\left(\mathfrak{M}_{(c)}\right)<\operatorname{Var} F_{k-1}\left(\mathfrak{M}_{(c)}\right)(4 \leqq k \leqq c)$, observe by Lemma 5.1 again that

$$
w_{c-1 . k-1}=\left[w_{k-1}, x_{k}, \cdots, x_{c-1}\right]
$$

is not a law in $F_{k-1}\left(\mathfrak{M}_{(c)}\right)$ but is clearly a law in $F_{k-2}\left(\mathfrak{M}_{(c)}\right)$. This completes the proof of the theorem.

\section{Concluding remarks}

Let $F$ be a free group of finite or countably infinite rank and let $W$ be a fully invariant subgroup of $F$. In general the centre of $F /[W, F]$ is not $W /[W, F]$ (see Cossey [3] for an example). If $W=F^{\prime \prime}$, then using Lemma 2.1, it is not difficult to see that the centre of $F /\left[F^{\prime \prime}, F\right]$ is precisely $F^{\prime \prime} /\left[F^{\prime \prime}, F\right]$. Here we are able to prove the corresponding result for $W=\left[F^{\prime \prime}, F\right]$.

THEOREM 6.1. The centre of $F /\left[F^{\prime \prime}, F, F\right]$ is precisely $\left[F^{\prime \prime}, F\right] /\left[F^{\prime \prime}, F, F\right]$.

ProOF. Since $F^{\prime \prime} /\left[F^{\prime \prime}, F\right]$ is the centre of $F /\left[F^{\prime \prime}, F\right]$, it follows that the centre of $F /\left[F^{\prime \prime}, F, F\right]$ is contained in $F^{\prime \prime} /\left[F^{\prime \prime}, F, F\right]$. Let $w \in F^{\prime \prime} \mid\left[F^{\prime \prime}, F\right]$ such that $\left[w, x_{k}\right] \in$ $\left[F^{\prime \prime}, F, F\right]$ for all $k=1,2, \cdots$. By Lemma 4.2 (iii), $0=\alpha_{41}\left[w, x_{k}\right]=-\lambda_{43}^{k} \alpha_{31}(w)$. Thus $\alpha_{31}(w)=0$ and by Lemma 3.3, $w$ is a product of the form (10). Since $\left[w, x_{k}\right]$ is a law in $F /\left[F^{\prime \prime}, F, F\right]$, it follows by (proof of) Theorem 4.6 that $\left[u_{1234}(x), x_{4}\right]$ is a law in $F /\left[F^{\prime \prime}, F, F\right]$, contrary to Theorem 4.1. Thus $w \in F^{\prime \prime} \backslash\left[F^{\prime \prime}, F\right]$ implies that $\left[w, x_{k}\right] \notin\left[F^{\prime \prime}, F, F\right]$, and hence the centre of $F /\left[F^{\prime \prime}, F, F\right]$ is precisely $\left[F^{\prime \prime}, F\right] /$ $\left[F^{\prime \prime}, F, F\right]$.

\section{References}

[1] M. Auslander and R. C. Lyndon, 'Commutator subgroups of free groups', Amer. J. Math. 77 (1955), 929-931.

[2] Gilbert Baumslag, 'Some theorems on the free groups of certain product varieties', J. Combinatorial Theory 2 (1967), 77-99.

[3] John Cossey, 'On decomposable varieties of groups', J. Austral. Math. Soc .11 (1970), 340342.

[4] Chander Kanta Gupta, 'A faithful matrix representation for certain centre-by-metakelian groups', J. Austral. Math. Soc. 10 (1969), 451-464.

[5] Chander Kanta Gupta, 'The free centre-by-metabelian groups', J. Austral. Math. Soc. 16 (1973), 294-299.

[6] Frank Levin, 'Generating groups for nilpotent varieties', J. Austral. Math. Soc. 11 (1970), 28-32. 
[7] Hanna Neumann, Varieties of groups. (Springer-Verlag, New York 1967).

[8] Ada Peluso, 'A residual property of free groups', Comm. Pure Appl. Math. 19 (1966), 435437.

[9] M. R. Vaughan-Lee, 'Generating groups of nilpotent varieties', Bull. Austral. Math. Soc. 3 (1970), 145-154.

University of Manitoba

Winnipeg, Canada

Rutgers, The State University

New Brunswick, N. J., U. S. A.

and

Ruhr Universität

Bochum, W. Germany. 